\title{
The importance of rapid diagnosis for multidrug-resistant Candida: an interview with Maurizio Sanguinetti
}

\author{
Maurizio Sanguinetti*,1 \\ ${ }^{1}$ Professor of Microbiology, Chief of the Department of Laboratory Sciences \& Infectious Diseases, Fondazione Policlinico \\ Universitario A. Gemelli, Rome, Italy \\ *Author for correspondence: Maurizio.sanguinetti@unicatt.it
}

\begin{abstract}
"we must stratify specific patient populations to find those most at risk of fungal infection and target them for resistance analysis with rapid tests"
\end{abstract}

In this exclusive interview, Maurizio Sanguinetti discusses current issues with Candida fungal infection diagnoses, in light of its rising resistance to antifungal drugs. This interview was conducted by Ellen Colvin, Editor of Future Microbiology.

Maurizio Sanguinetti, MD, is full Professor of Microbiology at the Università Cattolica del Sacro Cuore of Rome, Italy, and Director of the Institute of Microbiology and Chief of the Department of Laboratory Sciences and Infectious Diseases Sciences at the Fondazione Policlinico Agostino Gemelli IRCCS of Rome, Italy. For several years, the research activity of Maurizio Sanguinetti has mainly focused on the development of molecular methods for the rapid diagnosis of bacterial, mycobacterial and fungal infections; the elucidation of virulence and antimicrobial resistance mechanisms in clinically relevant bacterial and fungal pathogens; the characterization of the human microbiota in relationship to infectious and noninfectious diseases and implementation of new diagnostic strategies for the personalized care of patients with infectious diseases.

First draft submitted: 15 April 2019; Accepted for publication: 27 June 2019; Published online: 30 August 2019

Keywords: Aspergillus $\bullet$ azoles $\bullet$ Candida $\bullet$ echinocandins $\bullet$ MALDI-TOF mass spectrometry $\bullet$ rapid diagnostics $\bullet$ T2 magnetic resonance

\section{What is your research focus? What drew you to this area?}

My area of work is primarily medical mycology, with a focus on the implementation of new diagnostics that allow rapid testing of invasive fungal infections such as Candida and Aspergillus. Additionally, from a basic research perspective, my team and I are focusing on identifying genes involved in the virulence and drug resistance of pathogenic yeasts. In my hospital, and from an epidemiological point of view, we currently assess and monitor the emergence of antifungal drug resistance in these pathogenic yeasts.

\section{Why is rapid diagnosis of drug-resistant Candida important? What rapid diagnostics are available?}

Today, there are two main urgencies about the presence of drug-resistant Candida in hospital wards. One is the emerging multidrug resistance (MDR) in Candida glabrata, which has a lower susceptibility to azoles and a steadily increasing resistance to echinocandins. To tackle this, the tactic is to rapidly diagnose the possible $C$. glabrata MDR to establish a correct and accurate treatment as soon as possible. The second urgency concerns Candida auris, which is a well-known pathogen. The problem with $C$. auris is that this Candida species is naturally very resistant in the environment and as such, it is less susceptible to several classes of antifungal drugs. In practice, C. auris is extremely resistant to fluconazole and can be less susceptible to echinocandins and, in some cases, even to amphotericin B.

We therefore need to set up tests to rapidly diagnose the antifungal drug resistance, particularly for detecting the echinocandin resistance in C. glabrata and the antifungal resistance to echinocandins and azoles (other than

Future 8 Medicine 
fluconazole) in C. auris. Currently available tests for the rapid diagnosis of antifungal drug resistance include a molecular-based testing approach, which is very effective for detecting echinocandin resistance but is weaker for detecting azole resistance. This is because there are multiple mechanisms to determine azole resistance in different species of Candida, whereas the echinocandin resistance is only due to mutations in specific regions of the FKS1 and FKS2 genes and as such, it is easier to detect at a molecular level. Thus, contrary to molecular-based PCR diagnostic platforms, phenotypic-based approaches require the Candida strain be available in culture before assessing its antifungal susceptibility with a rapid test. For example, it is now possible to use MALDI-TOF mass spectrometry; thanks to the availability of the MALDI BioTyper antibiotic susceptibility test rapid assay (MBT-ASTRA), a commercial test that allows assessing antifungal drug resistance in both the C. glabrata and C. auris yeasts. To date, these are the two major approaches for the rapid detection of fungal resistance in clinical microbiology laboratories.

\section{How can this be translated to clinicians? How long do you think this will this take?}

The molecular PCR-based tests could potentially be rapidly translated into clinical practice, as some of the techniques involved are already used frequently. Additionally, there is hope for this as, although there are no commercial tests currently available, published work surrounding the ability of several of these diagnostic tools is very convincing. Thus, for in-house tests, it should be possible to implement them almost immediately when they become available. Of course, these are from positive blood cultures or similar and as such, it would be important to keep working toward improving these systems with the aim of being able to test the primary sample directly, in other words, the blood, in a similar manner to the T2 magnetic resonance (T2MR)-based system, but this is a goal for the future.

In my lab, in specific cases, we have been able to assess antifungal drug susceptibility from positive blood cultures using the MALDI-TOF mass spectrometry technology. This could be very important for clinical microbiology reports that show the results of susceptibility or not, for a specific case.

\section{What do you hope to see in the future? What will you work on next?}

For Candida, the currently available test used relies on the well-established T2MR system. The problem is the high expense, meaning it is difficult to widely implement; this is an issue for rapid diagnostic tests generally. To overcome this, we must stratify specific patient populations to find those most at risk of fungal infection and target them for resistance analysis with rapid tests. In my lab, we currently use $\beta$-glucan for diagnosis of candidemia; however, I believe we should use rapid T2MR-based tests on the $\beta$-glucan-positive patient population since patients are often false positive for $\beta$-glucans and this is an intrinsic problem. I personally want to make a screening for $\beta$-glucan, eliminating the negative patients, and in the positive patients, using the T2MR-based test to confirm $\beta$-glucan positivity.

\section{Disclaimer}

The opinions expressed in this interview are those of M Sanguinetti and do not necessarily reflect the views of Future Medicine Ltd.

\section{Financial \& competing interests disclosure}

The author has no relevant affiliations or financial involvement with any organization or entity with a financial interest in or financial conflict with the subject matter or materials discussed in the manuscript. This includes employment, consultancies, honoraria, stock ownership or options, expert testimony, grants or patents received or pending, or royalties.

No writing assistance was utilized in the production of this manuscript. 